\title{
A RAPID ONLINE TELEPATHY TEST ${ }^{1,2}$
}

RUPERT SHELDRAKE

Perrott-Warrick Project, London

\section{ASHWIN BEHAREE}

Department of Computer Science

University College, London

\begin{abstract}
Summary. - In an automated online telepathy test, each participant had four senders, two actual and two virtual, generated by the computer. In a series of 12 30 -sec. trials, the computer selected one of the senders at random and asked him to write a message to the subject. After $30 \mathrm{sec}$., the participant was asked to guess who had written a message. After the computer had recorded his guess, it sent him the message. In a total of 6,000 trials, there were 1,559 hits $(26.7 \%)$, significantly above the chance expectation of $25 \%$. In filmed tests, the hit rate was very similar. The hit rate with actual senders was higher than with virtual senders, but there was a strong guessing bias in favour of actual senders. When high-scoring subjects were retested, hit rates generally declined, but one subject repeatedly scored above chance.
\end{abstract}

The commonest kinds of seemingly telepathic experience occur in connection with telephone calls. Most people claim to have thought of someone for no apparent reason and then received a call from that person (Sheldrake, 2003). Skeptics routinely dismiss this phenomenon as a result of chance coincidence combined with selective memory (e.g., Marks, 2000): people remember when someone rings soon after being thought about, creating an illusion of telepathy, but forget when their thoughts about others are not followed by a call.

This selective memory hypothesis has now been tested experimentally. The procedure typically involves four potential callers. For each trial, one of the callers is selected at random, and is asked to call the subject. The randomly selected caller then telephones the subject, who has to guess who is calling before answering the phone. The details of all trials are recorded, so there is no possibility of selective memory. The probability of being right by chance is 1 in 4 , or $25 \%$.

Hundreds of telephone tests of this kind have shown positive, statistically significant hit rates (Sheldrake \& Smart, 2003a, 2003b; Lobach \& Bierman, 2004; Sheldrake, Godwin, \& Rockell, 2004). In filmed experiments in which participants were videotaped continuously, the average hit rate

\footnotetext{
${ }^{1}$ Address correspondence to Rupert Sheldrake, 20 Willow Road, London NW3 1TJ, UK or e-mail (rsheldrake@clara.co.uk).

${ }^{2}$ This work was supported by the Perrott-Warrick Scholarship, administered by Trinity College, Cambridge; by a grant from the Institute of Noetic Sciences, Petaluma, California; and by the generosity of Mr. Addison Fischer of Naples, Florida. We thank Gregory Gibbs, Mary Anne Kae, Robert Kenney, Ruth Kidson, Brenda Lau, Monica Liu, Sophie Newton, Ann Ryan, and Logan Yonavjak for their help in recruiting and testing participants.
}

DOI 10.2466/PR0.104.3.957-970 
was $45 \%$, compared with $25 \%$ expected by chance $\left(p<10^{-11}\right.$; Sheldrake \& Smart, 2003a). In similar tests with e-mails, the hit rates were also significant. In filmed e-mail experiments, the hit rate was $47 \%$, significantly above chance levels $\left(p=10^{-7}\right.$; Sheldrake \& Smart, 2005).

These telephone and e-mail telepathy tests were organized by experimenters who carried out randomizations and gave instructions to the callers or e-mailers one trial at a time. In a new procedure, developed by Sheldrake and Lambert (2007), telepathy tests were automated and carried out online. As in the telephone and e-mail telepathy tests, the participant had to guess which of four potential senders was sending him a message. The test was designed to take place rapidly, and each of the 10 trials in a test lasted only one minute. In a total of 1,980 trials, there were 581 hits $(29.3 \%)$, significantly above the chance expectation of $25 \%$. In some tests, there were only two actual senders and two virtual senders, generated by the computer. There were higher hit rates with actual than virtual senders, with whom no telepathy would be possible.

The present paper describes a new online telepathy test in which the procedure was even more rapid, with each trial lasting only $30 \mathrm{sec}$. In all tests there were two actual and two virtual senders. The use of virtual senders inevitably diluted any possible telepathic effect, but previous research had shown that reducing the number of actual senders from four to two made it easier to find participants. With two virtual senders, the maximum hit rate that a perfectly telepathic subject could achieve would be $75 \%$. He would be able to identify each of the actual senders and also identify when there was no actual sender. But he would not be able to distinguish between the two virtual senders and would have to guess. The purposes of the present experiment were, first, to replicate the previous online telepathy test on a more extensive scale, with 6,000 trials as compared to fewer than 2,000 and, second, to investigate whether telepathy could be studied effectively through an even more rapid procedure.

An obvious advantage of an online test over laboratory-based studies is that the internet-based procedure enables a wide range of people to participate in many different locations. It facilitates the investigation of variables such as the ages of subjects, their relationships to senders, and the distance between senders and subjects, who can be thousands of miles apart. On the other hand, it is more difficult to supervise the tests and hence to rule out cheating. There is no evidence from previous automated telepathy tests, or from nonautomated telephone and e-mail telepathy tests, that any of the participants were in fact cheating, but this possibility cannot be ignored. However, automated online tests can be carried out under rigorous, videotaped conditions, as shown in this paper. 


\section{Methods}

\section{Programming}

The test was based on a previous procedure programmed by Michael Lambert with 101 -min. trials per test (Sheldrake \& Lambert, 2007) but the coding was modified by the second author in such a way that the test consisted of a series of 1230 -sec. trials in which the computer selected one of four potential senders at random. Two were actual people nominated by the receiver, and two were virtual senders generated by the computer, called Virtual Sender 1 and Virtual Sender 2.

The coding was carried out in Hypertext Preprocessor Protocol (PHP Version 4.4.4-8), an HTML-embedded scripting language widely used on the internet for generating web pages dynamically, often using a database for the source data. Randomization for the experiment was provided by the system-level randomizer supplied with the Linux operating system running on the web server. This randomizer is technically represented by the /dev/random 1 device, and generates random numbers based on an "entropy pool" of random numbers. New randomness was added to this pool when unpredictable events happened, such as the pressing of a key by the user at a particular time. This randomizer was used to select the sender at each trial. Data were stored on a MySQL Version 5.0 database, which could be accessed online by the experimenter with the use of a password.

\section{Procedure}

To carry out the test, a participant had to register with the group in advance, choosing a group name and a password. When registering, participants gave their own name, sex, age, city and country of residence, and e-mail address. Participants also provided the name, sex, age (within a preset age range, such as 10-14, 15-19, 20-29, 30-39, etc.), and e-mail address of each sender, also stating each sender's relationship to them (e.g., friend, mother, colleague) and the approximate geographical distance between the participant and the sender. They also gave their estimate of which sender they were "most likely to be telepathic with." In order to do the test, the participant and the senders logged on to the experiment at the same time. They did this through the web site of the first author, using their group name and password that had been registered in advance.

In a series of 12 trials, each lasting 30 sec., one of the four senders was selected at random and asked by the computer to compose a message for the target participant in a special message box. At the end of the trial period, the sender sent this message to the computer. The participant was then asked which of the four senders (including the two virtual senders) he thought the message was from. All four names were presented (in the 
same order each time) and he indicated his guess by clicking beside one of these names. After he had made his guess, the message was delivered, and hence he received immediate feedback as to whether the guess was a hit or not. Another trial then began. When all 12 trials had been completed, all the participants received a message giving the number of hits.

Participants were given the following instructions prior to registering: "How telepathic are you? Have you ever had the experience of knowing who's on the phone before you pick it up? Or you think about someonemaybe for the first time in a while-and soon afterwards an e-mail arrives? We want to find out if this is just a matter of coincidence, or whether telepathy is involved. All you have to do is guess which one of four people is sending you a message. By the laws of chance, you'll be correct about $25 \%$ of the time, but our findings in similar experiments show that some people are correct much more often than that, which suggests that telepathy is involved. Are you one of these people?" The experimental procedure was explained as follows: "The experiment involves a subject (you) and two senders. You know that you will get a message at a fixed time, and you know it will be from one of these senders, or from one of two virtual senders generated by the computer. The sender is picked at random. Just before the message is due to be sent to you, you will be asked to guess who it is going to be from. You do this twelve times. The whole thing takes 15-20 minutes."

The general instructions to the participants registering on the web site were as follows: "Choose your experimental group. This will consist of yourself and two close friends or family members-your senders. You are more likely to be successful if you choose people you are close to emotionally, but physical distance is not a problem. They can be anywhere in the world. The computer will generate two virtual people to make the number up to four. Choose a time (immediately or in the future) when everyone can be available at the computer for up to 20 minutes. Register the details of your experimental group online at www.sheldrake. org. You and they log on at the agreed time, making sure that everyone's sound system is activated. For each of the trials, the computer will pick one of the senders at random and will ask them to think about you and write you a message. You will be alerted when you are about to receive a message, and you indicate who you think is about to send it. You will either be right or wrong. (You will probably do better if you don't think too much about it and go with the first thought that comes to you.) After all 12 trials, you will be given your results in a form which you can print out if you wish."

Most of the participants were recruited by work scholars in the UK and USA. Several of the work scholars were 17- and 18-year-old students at 
a girls' high school in London, and as a result there were more female than male participants. A summary of the results of all tests, both complete and incomplete, was accessible online to the experimenter, with the use of a password. The experiment was terminated at a predetermined point when the number of complete tests reached 500, giving a total of 6,000 trials.

\section{Videotaped Tests}

Some participants who had achieved hit rates above chance were retested under videotaped conditions. The experimenter set up the camera in such a way that the subject and the computer screen were in full view. Before the filming began, subjects were asked to turn off their cell phones and any instant messaging systems and they were videotaped continuously throughout the experiment. All videos were dated and time-coded. Having set up the video camera, the experimenter left the room, and made sure than no one else entered it while the test was in progress.

The videos were evaluated by the first author's research assistant, who was given no details about the subjects or their hit rates. In the evaluation she checked to see if at any stage the subjects went off-camera, received telephone calls, text messages, instant messages, or if anyone entered the room. She found no evidence in any of the tests of possible cheating by these means.

\section{Statistical Analysis}

The data were analysed by the exact binomial test; the expected probability of a hit by chance was .25. The null hypothesis was that hit rates would be at the chance level. One-sided tests were used. The comparison of data from different groups (e.g., male and female subjects) was carried out using the $2 \times 2$ chi-squared test (Campbell, 1989). Cohen's effect size $d$ was calculated according to the formula $d=p$ (hits observed) $-.25 /$ square root $(.25 \times .75)$.

\section{Results}

\section{Overall Results}

In the 500 completed tests of 12 trials each, there were 1,599 hits out of 6,000 trials $(26.7 \%)$, significantly above the chance level of $25 \%(p=.002)$. The $95 \%$ confidence interval of this hit rate is from 26 to $28 \%$. The effect size in terms of Cohen $d$ was 0.03 .

In the incomplete tests, there were 118 trials altogether, with 26 hits $(22.0 \%)$, not significantly different from the $25 \%$ chance level. When all trials were included, complete and incomplete, the overall hit rate was $26.6 \%$ $(p=.005)$. In the following analysis of results, only data from complete trials were included. 


\section{Supervised Tests}

Some tests were videotaped, and the films were evaluated by an independent researcher to make certain that participants were not receiving any e-mails, text messages, instant messages, or other communications during the test sessions. In these filmed tests the hit rate was 128 out of 468 trials $(27.3 \%)$, similar to the hit rate in the unfilmed tests.

\section{Actual and Virtual Senders}

In the 500 completed tests, there were approximately equal numbers of trials with actual and virtual senders. The hit rate with actual senders was $33.7 \%$, significantly higher than the hit rate with virtual senders of $19.5 \%$ (see Table 1 ). There was a strong guessing bias in favour of the actual senders: in 3,775 out of $6,000(62.9 \%)$ trials the subjects guessed the names of actual senders, while the senders were actual in only $50.2 \%$ of the trials. When the hit rates were expressed on the basis of guesses rather than trials, they were almost the same: $26.9 \%$ with actual senders and $26.2 \%$ with virtual senders. This difference was not statistically significant.

TABLE 1

Numbers of Hits With Actual and Virtual Senders Expressed as a Percentage of Trials and of Guesses. Results From 500 Tests With 6,000 Trials

\begin{tabular}{cccccc}
\hline \hline Senders & Trials & Guesses & Hits & Hits $(\%$ trials $)$ & Hits $(\%$ guesses $)$ \\
\hline Actual & 3,016 & 3,775 & 1,017 & 33.7 & 26.9 \\
Virtual & 2,984 & 2,225 & 582 & 19.5 & 26.2 \\
\hline
\end{tabular}

Sex and Age of Subjects

There were 162 tests with male participants, with 502 hits out of 1,944 trials (25.8\%), and 338 tests with female participants, with 1,097 hits out of 4,056 trials $(27.0 \%)$. Thus, the hit rate with female subjects was slightly higher, but this difference was not significant statistically.

Subjects' ages ranged from below 10 to 69 . The number of trials and hit rates with the different prespecified age groups are shown in Table 2. The hit rate was highest in the 15- to 19-yr.-old group, significantly higher than in the 20- to 29-yr.-old group. The hit rate was lowest in the 30- to 39yr.-old group, where it was at the chance level.

\section{Participants' Predictions of Success}

When registering for the test, participants indicated with which of their two senders they were more likely to be telepathic. When their hits were expressed on the basis of trials, they were indeed slightly more successful with those they ranked more likely, with a hit rate of 518 out of 1,496 trials (34.6\%) as opposed to 495 out of 1,509 trials (32.8\%) with the "less likely" senders, but this difference was not statistically significant. They also showed a guessing bias towards the "more likely" senders. When the hit 
TABLE 2

Hit Rates of Participants by Age Group

\begin{tabular}{crrc}
\hline \hline Age & Trials & Hits & Hits \% \\
\hline $10-14$ & 228 & 60 & 26.3 \\
$15-19$ & 1,968 & 550 & 27.9 \\
$20-29$ & 696 & 182 & 26.1 \\
$30-39$ & 768 & 193 & 25.0 \\
$40-49$ & 1,032 & 271 & 26.3 \\
$50-59$ & 900 & 236 & 26.2 \\
$60-69$ & 408 & 108 & 26.5 \\
\hline
\end{tabular}

rates were expressed on the basis of guesses, the difference was even smaller: $518 / 1,896$ guesses or $27.3 \%$ with the more likely and $495 / 1,863$ or $26.6 \%$ with the less likely. Again this difference was not significant.

Relationships Between Senders and Participants

The effects of different sender-participant relationships are shown in Table 3. In all cases there was a guessing bias in favour of the sender, so the hit rates were lower when expressed on the basis of guesses as opposed to trials. On the basis of guesses, the highest hit rates were with mothers, spouses, and other family members (a category that included cousins, aunts, nephews, etc.). Overall there were slightly higher hit rates with family members than with people who were not family members, but these differences were not statistically significant.

TABLE 3

Hit Rates of Participants by Relationship to Sender

\begin{tabular}{lrcc}
\hline \hline \multicolumn{1}{c}{ Sender } & Trials & Hits (\% trials) & Hits (\% guesses) \\
\hline Mother & 157 & 34.3 & 28.0 \\
Father & 53 & 41.5 & 26.1 \\
Sister & 146 & 34.2 & 27.2 \\
Brother & 110 & 36.4 & 25.0 \\
Daughter & 112 & 32.1 & 25.7 \\
Son & 75 & 26.6 & 22.9 \\
Spouse & 143 & 33.6 & 28.4 \\
Other family & 248 & 34.6 & 29.5 \\
Family total & 1,053 & 34.1 & 27.2 \\
& & & \\
Friends & 1,539 & 34.2 & 26.9 \\
Colleagues & 194 & 33.0 & 25.5 \\
Others & 230 & 29.6 & 27.8 \\
Nonfamily total & 1,963 & 33.5 & 26.8 \\
\hline
\end{tabular}

\section{Effects of Distance}

The effects of the distance between sender and participant are shown in Table 4. In all cases the hit rates on the basis of guesses were lower than on the basis of trials, because of the general response bias in favour of ac- 
tual as opposed to virtual senders. The highest hit rates were those at the greatest distances, over 500 miles, and the next highest were those when senders and participants were closest. At intermediate distances, between 11 and 500 miles, the hit rates on the basis of guesses were slightly below the chance level. On the basis of guesses, the hit rates were significantly higher $(p=.04)$ for distances over 500 miles than for distances between 11 and 500 miles.

TABLE 4

Participants' Hit Rates by Distance From Sender

\begin{tabular}{lrcc}
\hline \hline Miles & Trials & Hits $(\%$ trials $)$ & Hits $(\%$ guesses $)$ \\
\hline$<1$ & 1,426 & 35.0 & 27.8 \\
$1-10$ & 652 & 33.7 & 26.8 \\
$11-50$ & 267 & 27.3 & 24.2 \\
$51-100$ & 170 & 31.2 & 23.7 \\
$101-500$ & 140 & 30.0 & 22.3 \\
$501-1000$ & 180 & 34.4 & 30.0 \\
$>1001$ & 181 & 37.6 & 28.5 \\
\hline
\end{tabular}

Repeated Tests

Some subjects spontaneously carried out the test more than once; and some of those who had been recruited by work scholars were asked to do the test again to study the effect of repeated testing, especially if they had relatively high hit rates. The hit rates in repeated tests are shown in Table 5. The high hit rate in the first test reflects the fact that there was a preponderance of high-scoring participants in this sample. When tested a second time, the average hit rate dropped from 45.2 to $35.2 \%$, but it was still highly significant statistically. However, by the third test, the hit rate had dropped to the chance level, and in the fourth test it was below chance, although not significantly so.

TABLE 5

Hit Rates with Repeated Testing

\begin{tabular}{cccc}
\hline \hline Test & Trials & Hits & Hits \% \\
\hline 1 & 372 & 168 & $45.2 \dagger$ \\
2 & 372 & 131 & $35.2^{*}$ \\
3 & 131 & 32 & 24.4 \\
4 & 84 & 13 & 15.5 \\
\hline
\end{tabular}

${ }^{{ }^{*}} p=10^{-5} \cdot+p=10^{-7}$.

\section{An Unusually Sensitive Subject}

In the prototype version of this experiment (Sheldrake \& Lambert, 2007), one of the subjects, AF, a 15-year-old girl attending school in North London, had an unusually high hit rate. With two actual and two virtual senders she had a total score of $17 / 25(68 \%)$, significantly above the chance 
level of $25 \%\left(p=10^{-5}\right)$. AF described herself thus, "I have been quite familiar with psychic phenomena from a young age due to the influence of my mother, who always had a strong interest in the subject. My mother and myself [sic] would often practise various psychic mind-reading exercises."

AF took part in the experiment described in this paper. In her first test her hit rate was $8 / 12$. In response to asking her how she felt during the test, she replied, "The only way I could tell it was a virtual sender was when I felt no incoming energy from either of the two individuals involved. But selecting either virtual sender 1 or 2 was a matter of guessing." AF subsequently took part in a further series of tests, supervised and filmed in her family home. The hit rate in these tests was $17 / 36$ (47\%, as opposed to $25 \%$ by chance, $p=.003$ ). She also took part in three control tests, in which there were no actual senders, with a hit rate not significantly different from chance. See Table 6 for these results.

TABLE 6

Results for Participant AF with Actual and Virtual Senders in an Unfilmed Test, Three Filmed Tests, and Three Control Tests with No Actual Senders

\begin{tabular}{|c|c|c|c|c|c|c|c|c|}
\hline \multirow[t]{2}{*}{ Test } & \multicolumn{2}{|c|}{ Real } & \multicolumn{2}{|c|}{ Virtual } & \multicolumn{4}{|c|}{ Total } \\
\hline & Trials & Hits & Trials & Hits & Trials & Hits & Hits $\%$ & $p$ \\
\hline Unfilmed & 3 & 3 & 9 & 5 & 12 & 8 & 66 & .003 \\
\hline Filmed 1 & 4 & 2 & 8 & 1 & 12 & 3 & & \\
\hline Filmed 2 & 5 & 3 & 7 & 3 & 12 & 6 & & \\
\hline Filmed 3 & 5 & 3 & 7 & 5 & 12 & 8 & & \\
\hline Filmed Total & 14 & 8 & 22 & 8 & 36 & 17 & 47 & .003 \\
\hline Control 1 & 5 & 0 & 2 & 1 & 7 & 1 & & \\
\hline Control 2 & 3 & 1 & 9 & 4 & 12 & 5 & & \\
\hline Control 3 & 7 & 2 & 5 & 1 & 12 & 3 & & \\
\hline Control Total & 15 & 3 & 16 & 6 & 31 & 9 & 29 & $n s$ \\
\hline
\end{tabular}

\section{Discussion}

In the 500 complete tests, the overall hit rate of $26.7 \%$ was significantly above the chance level of $25 \%(p=.002)$. When incomplete tests were added to this total, the overall hit rate of $26.6 \%$ was also significantly above the chance level ( $p=.005)$. The findings are not the result of "optional stopping" or the "file drawer effect," where only positive data are published. All the data from this test are reported. In a previous version of this online test, the hit rate was higher (29.3\%; Sheldrake \& Lambert, 2007). This difference between the two versions was significant at the $p=.02$ level, according to the $2 \times 2$ chi-squared test. The earlier and the present versions of the test differed in two main ways. (1) In the present test, all subjects had only two actual senders and two virtual senders. In the previous version, participants had up to four actual senders. Since telepathy by defini- 
tion involves mind-to-mind communication, it is not possible with virtual senders generated by the computer. So the use of two virtual senders inevitably diluted the effect. Even if a participant could feel from the absence of telepathy that neither of the actual senders was involved, and hence that there must be a virtual sender, he could not feel which virtual sender was involved. The choice between virtual senders could therefore be only a matter of guessing, as the most sensitive participant, AF, explicitly observed. (2) In the previous version, there were 10 trials per test, each lasting $1 \mathrm{~min}$. In this version, there were 12 trials lasting $30 \mathrm{sec}$. each, so the test proceeded faster. It is possible that this procedure was too fast both for subjects and for senders. Senders were asked not to think about the subject until requested to do so, and then after the 30-sec. trial period to switch off again. This requirement may have been unrealistic. Senders had to continue to pay attention to the instructions on the screen to see if they were selected for a new trial. Because they were so continuously involved in the test, most senders may not have been able to empty their minds and stop thinking about the subject in trials in which they were not selected. The greater frequency of trials in this new version of the test required even more sustained attention. If senders who were not selected for a given trial were nevertheless thinking about the test, and hence about the subject, then the subject may have received confusing telepathic influences from both of the actual senders whether or not they had been selected. This is an inherent problem in any telepathy test that requires the continuous attention of senders, and it may have been exacerbated by speeding up the procedure.

In previous telepathy tests involving telephone calls and e-mails, the effect sizes were much greater. In videotaped tests with telephone calls, the effect size, expressed in terms of Cohen $d$, was 0.46 (Sheldrake \& Smart, 2003b) and in videotaped tests with e-mails 0.50 (Sheldrake \& Smart, 2005). In the present experiment, Cohen $d$ was only 0.03. One reason for this small effect size could be that the senders were required to pay continuous attention to the test. In the telephone and e-mail telepathy tests, there were intervals of $10 \mathrm{~min}$. between trials, and senders were not required to pay attention to the test until they were needed. They simply continued with their normal life until they received a telephone call or email asking them to take part in a trial. They then had several minutes to think about the subject before calling or e-mailing him.

Possibility of Cheating

Most of the tests were unsupervised, and hence it is conceivable that some participants could have cheated. First, the subject and one or more of his senders could have been in the same room and the senders could have told the subject when they were selected. Secondly, one or both of 
the senders could have informed the subject when they were selected by instant messages, or by e-mail, or by telephone. However, cheating was probably minimal. Participants had no motive or incentive to cheat. Moreover, if cheating were widespread, then the overall hit rates likely would have been much higher. Nevertheless, to control for this possibility, some of the tests were supervised and videotaped in such a way that any of these forms of cheating would have been detected, but in no case was there any evidence of cheating, and the hit rates were similar to those in unsupervised tests. Also, in the case of the highest-scoring participant, the hit rates in filmed trials were still significantly above the chance level. Hence, although one cannot rule out the possibility that a few people cheated in unsupervised tests, this cannot be a full explanation for the above-chance hit rates in this experiment.

\section{Actual and Virtual Senders}

There was a strong guessing bias in favor of actual senders (Table 1), which in itself raised the hit rates with actual senders and reduced those with virtual senders. In the most extreme case, if a subject always guessed the name of an actual sender he would have a hit rate of $0 \%$ with virtual senders, and, by pure guessing, a hit rate with real senders of $50 \%$. The overall hit rate would of course be at the chance level of $25 \%$. In fact, in $62.9 \%$ of the trials, the subjects guessed the name of a real sender, while only $50.2 \%$ of the trials actually had real senders. To correct for this guessing bias, the hit rates were expressed on the basis of guesses rather than trials. On this basis there was no significant difference between the hit rate with real $(26.9 \%)$ and virtual $(26.2 \%)$ senders. A similar response bias occurred in the earlier version of this automated online experiment in tests with two real and two virtual senders (Sheldrake \& Lambert, 2007).

At first sight, any above-chance hit rate with virtual senders seems unlikely in a telepathy test, since telepathy would not be possible with these nonpeople. However, if a subject can detect real senders telepathically, then it should also be possible to detect the absence of a real sender telepathically, since in this situation absence of telepathy is itself a kind of telepathy. But with two virtual senders, a perfectly telepathic participant could not distinguish between the two. Thus, as discussed in the Introduction, a perfectly telepathic subject would score $100 \%$ with real senders and $50 \%$ with virtual senders, with an overall hit rate of $75 \%$, as opposed to $25 \%$ by chance. In some of her tests, AF approached this theoretical maximum (Table 6).

\section{Characteristics of Senders and Subjects}

There was no significant effect for sex of participant. The highest hit rates were found among 15- to 19-yr.-olds (Table 2). There were higher hit 
rates when senders and participants were family members (Table 3), but this effect was not statistically significant. Subjects had higher hit rates with the senders they thought they were more likely to be successful with, but they also had a stronger response bias towards these senders, and when this was taken into account the difference was not significant. These findings are in general agreement with the results of the earlier version of this experiment (Sheldrake \& Lambert, 2007).

The highest hit rates occurred when the senders and subjects were furthest apart, over 1,000 miles (Table 4). Again, a similar effect was found in the earlier version this experiment (Sheldrake \& Lambert, 2007). The available evidence suggests that apparent telepathic communication does not decline with distance (Sheldrake, 2003), but it would be surprising if it increased. A possible explanation for this effect could be that subjects chose senders who were far away only if these were people they knew well, such as family members. Telepathy seems to occur most effectively between people who know each other well (Sheldrake, 2003), and closeness of relationship may be a more important factor than physical proximity.

\section{Effects of Repeated Testing}

When high-scoring subjects were tested again, their hit rates fell from 45.2 to $35.2 \%$, still very significantly above chance. But when tested for a third or fourth time, the hit rates were no better than chance (Table 5). By contrast, AF's scores showed no such general decline, although in the first of her filmed tests her hit rate was also at the chance level (Table 6). It is difficult to explain this decline. If the high scores in the first tests were simply due to chance, then the scores in the second tests should immediately have reverted to the chance level, but they did not. Instead, the results suggest that most of the participants progressively lost their previous sensitivity the more often they were tested. One explanation is that the subjects became more nervous or self-conscious when retested, and this may have interfered with their telepathic ability. The fact that AF's hit rate was at the chance level in her first filmed test may have a similar explanation: indeed, she said at the time that she was feeling nervous. But the difference between AF and the other subjects was that she had taken part in many previous telepathy tests with her mother and therefore was more experienced in functioning under the artificial conditions of randomized testing.

Telepathy and other psychic phenomena are often described as "elusive." For skeptics, this is an argument against their very existence: if the phenomena cannot be demonstrated repeatedly and on demand, then they should be treated with the utmost suspicion (Hyman, 1989). However, if one of the reasons for the elusiveness of psychic phenomena is that they are inhibited by nervousness or self-consciousness, then there seems to be two ways forward. One is to work with apparently sensitive subjects, 
like $\mathrm{AF}$, who are frequently tested; but such subjects are very rare. Another is to test people under relaxed conditions that give rise to relatively little nervousness or anxiety. The present experiment enables people to do the test at home in more relaxed conditions than in a laboratory. Yet the attempt to increase rigor through repeated testing and filming has run into the all-too-familiar problem of elusiveness. This problem is made worse by the small effect size. Telepathy tests that show larger effect sizes, such as tests with telephone calls and e-mails, have so far proved easier to repeat under more rigorous, filmed conditions.

\section{Telepathy or Other Kinds of Extrasensory Perception}

Parapsychologists distinguish between three kinds of extrasensory perception (ESP): telepathy, clairvoyance, and precognition (Wolman, 1977). The above-chance results observed in the present experiment may have been due to clairvoyance or precognition, rather than telepathy. If subjects were clairvoyant, they would have known which senders the computer had selected without the need for senders to think about the subjects, or indeed without the need for senders at all. If they were precognitive, they could have anticipated the message they were about to receive, and thus identified the sender in advance. The best way to distinguish between the effects of telepathy and other forms of ESP is to run control experiments without real senders, thus eliminating the possibility of telepathy but still leaving open the possibility of clairvoyance or precognition. Such control tests were done with AF (Table 6). The hit rates were not significantly different from chance, implying that AF's abovechance hit rates in tests with real senders were indeed more likely to result from telepathy rather than from clairvoyance or precognition.

\section{Further Research With Automated Tests}

This rapid, automated online telepathy test seems to have been overambitious. It has a lower effect size than the slower version of this test, suggesting that speeding up the test procedure is not helpful. But even the slower previous version, with trials just over $1 \mathrm{~min}$. apart, had a much lower effect size than yet slower tests, such as telephone and e-mail telepathy tests, with trials about $10 \mathrm{~min}$. apart. Perhaps slow tests have two advantages. First, they enable the participant to relax between trials and do something else, and second, they do not require the continuous involvement of senders, who can therefore get on with other tasks until they are selected to take part in a trial, which might be only after 30 or 40 minutes. In designing new automated telepathy tests, it will be better to space trials farther apart to enable the senders to detach themselves from the test when they are not required. Automated versions of telephone telepathy and e-mail telepathy tests would meet these criteria. 


\section{REFERENCES}

Campbell, R. C. (1989) Statistics for biologists. (3rd ed.) Cambridge, UK: Cambridge Univer. Press.

Hyman, R. (1989) The elusive quarry: a scientific appraisal of psychical research. Amherst, NY: Prometheus Books.

Lobach, E., \& Bierman, D. J. (2004) Who's calling at this hour? Local sidereal time and telephone telepathy. Proceedings of the Parapsychological Association Annual Convention, 91-97.

Marks, D. (2000) The psychology of the psychic. Amherst, NY: Prometheus Books.

SHELdRAKe, R. (2003) The sense of being stared at, and other aspects of the extended mind. London: Hutchinson.

Sheldrake, R., Godwin, H., \& Rockell, S. (2004) A filmed experiment on telephone telepathy with the Nolan sisters. Journal of the Society for Psychical Research, 68, 168-172.

Sheldrake, R., \& LAmbert, M. (2007) An automated online telepathy test. Journal of Scientific Exploration, 21, 511-522.

Sheldrake, R., \& Smart, P. (2003a) Experimental tests for telephone telepathy. Journal of the Society for Psychical Research, 67, 184-199.

Sheldrake, R., \& Smart, P. (2003b) Videotaped experiments on telephone telepathy. Journal of Parapsychology, 67, 187-206.

Sheldrake, R., \& SMART, P. (2005) Testing for telepathy in connection with emails. Perceptual and Motor Skills, 101, 771-786.

Wolman, B. B. (Ed.) (1977) Handbook of parapsychology. New York: Van Nostrand Reinhold.

Accepted April 27, 2009. 